\title{
Young plasma attenuates cognitive impairment and the cortical hemorrhage area in cerebral amyloid angiopathy model mice
}

\author{
Nan Li ${ }^{1 \#}$, Xu Zhang ${ }^{1 \#}$, Zhiqiang Gu ${ }^{2}$, Chunhe Su${ }^{1}$, Haojun Lian ${ }^{1}$ \\ ${ }^{1}$ Department of Neurology, The Second Affiliated Hospital of Zhengzhou University, Zhengzhou, China; ${ }^{2}$ Department of Radiology, The Second \\ Affiliated Hospital of Zhengzhou University, Zhengzhou, China \\ Contributions: (I) Conception and design: N Li; (II) Administrative support: Z Gu; (III) Provision of study materials or patients: N Li; (IV) Collection \\ and assembly of data: X Zhang, C Su; (V) Data analysis and interpretation: X Zhang, H Lian; (VI) Manuscript writing: All authors; (VII) Final \\ approval of manuscript: All authors. \\ "These authors contributed equally to this work. \\ Correspondence to: Nan Li. Department of Neurology, The Second Affiliated Hospital of Zhengzhou University, Zhengzhou, China. Email: linan@zzu.edu.cn.
}

Background: Cerebral amyloid angiopathy (CAA) is characterized by the deposition of $\beta$-amyloid (A $\beta$ ) in leptomeningeal vessels and penetrating arterioles. Intracerebral hemorrhage (ICH) is one of the most destructive complications in CAA. Young plasma has been shown to improve cognitive, learning, and memory functions in Alzheimer's disease (AD) model mice and is a new potential therapy. However, it is not clear whether young plasma can reduce cerebral hemorrhage and improve the prognosis of neurological function in APP/PS1 (which express APP695swe and PS1-dE9 mutations) mice with CAA disease.

Methods: The Y-maze, new object recognition (NOR), forced swimming, open field, sucrose consumption, and corner tests were used to evaluate the learning and memory, cognitive ability, and emotional changes in CAA model mice. The effect of young plasma on neurogenesis was analyzed by immunofluorescence. The level of $\mathrm{A} \beta$ in the cerebral cortex and hippocampus of mice was measured by enzyme-linked immunosorbent assay (ELISA). Finally, the area of cortical hemorrhage in mice was analyzed by fast blue-staining.

Results: We proved that young plasma improved cognition, learning and memory impairment, and anxiety in CAA model mice, prevented neuronal apoptosis, and enhanced neurogenesis in APP/PS1 mice. However, young plasma did not reduce the level of $\mathrm{A} \beta$ in the cortex and hippocampus of APP/PS1 mice. We also found that young plasma reduced the area of cerebral hemorrhage in APP/PS1 mice.

Conclusions: Our results show that young plasma can improve learning and memory, cognitive impairment, and anxiety in CAA model mice and can reduce the area of cortical hemorrhage.

Keywords: Cerebral amyloid angiopathy (CAA); young plasma; cortical hemorrhage; cognitive impairment; enzyme-linked immunosorbent assay (ELISA)

Submitted Nov 16, 2020. Accepted for publication Jan 08, 2021.

doi: 10.21037/atm-20-8008

View this article at: http://dx.doi.org/10.21037/atm-20-8008

\section{Introduction}

Cerebral amyloid angiopathy (CAA) is a cerebrovascular disease which manifests clinically as progressively aggravated cognitive dysfunction, transient focal neurological dysfunction, and repeated or multiple cerebral lobar hemorrhage (1-3). An autopsy shows that the incidence of CAA in the general elderly population is $10 \%$ to $40 \%$, and the incidence of CAA in Alzheimer's disease (AD) patients with Alzheimer's disease is as high as $80 \%$ (4). There was no significant gender difference in the incidence of CAA, but the incidence increased significantly with age. The incidence of CAA autopsy in $60-70$ years old is $33 \%$, and it is as high as $75 \%$ over 90 years old (5). Verbeek et al. (6) detected $\beta$-amyloid (A $\beta$ ) $40, \mathrm{~A} \beta 42$ and tau protein levels in normal people, $\mathrm{AD}$ and CAA patients, and the results showed that $A \beta 40$ and $A \beta 42$ 
in CAA cerebrospinal fluid were significantly reduced, while tau protein and phosphorylated tau protein levels were significantly increased, suggesting that cerebrospinal fluid $\mathrm{A} \beta 40, \mathrm{~A} \beta 42$, tau protein levels can be used as biological markers for advanced CAA sensitivity. Some studies have shown that there is a high risk of Apolipoprotein E (APOE) $\varepsilon 4$ allele developing into CAA with age (7). Most of CAA progressed to cerebral ischemia, accompanied by mental disorders and behavioral abnormalities, such as memory, dyscalculia and analysis, or hallucinations and delusions. Some patients showed nervous system abnormalities, such as dysphasia, clonus or generalized convulsions, and a few had hemiplegia. Severe cerebral hemorrhage occurs in patients with elevated blood pressure, accompanied by headache, nausea, vomiting and insanity. The characteristic neuroimaging changes of cerebral amyloid angiopathy include cerebral microbleeds (CMBs), white matter hyperintensity $(\mathrm{WMH})$, enlarged perivascular space [EPVS, also known as enlarged Virchow-Robin space (dVRS)] and cortex Hemosiderin deposits on the surface. In 2010, the University of Munich Hospital in Germany released a revised version of the CAA Boston standard (8). If the autopsy found hemorrhage in the cerebral lobe and cortex, and diagnosed severe amyloid angiopathy, but no other causes of cerebral hemorrhage, it can be confirmed as suffering from CAA. If the results of CT and magnetic resonance imaging (MRI) show that the hemorrhage is limited to a single focus of hemorrhage in the cerebral lobe or cortex or with a small amount of hemosiderin deposition on the surface of the cortex, there is a certain degree of amyloid angiopathy, the age of onset is $\geq 55$ years old, and there is no other cause of cerebral hemorrhage, then it is likely to be diagnosed as CAA. Currently, there is no effective treatment to prevent or reverse the deposition of amyloid. Clinically, it mainly treats the complications of CAA, such as dementia and recurrent cerebral hemorrhage, etc.

Among the clinical complications caused by CAA, cerebral hemorrhage is the most destructive. The clinical manifestations of CAA are partially similar to $\mathrm{AD}$, and $80 \%$ or more of CAA patients demonstrate $\mathrm{AD}$ pathological changes (9). Studies have shown that high-fat diet can enhance cerebral amyloidosis and cognitive impairment in $\mathrm{AD}$ model mice and significantly promote the development of AD-like pathology by enhancing cerebral amyloidosis and oxidative stress which has nothing to do with metabolic disorders (10).CAA is a cerebral vessel disease caused by A $\beta$ deposition at the vessel walls $(2,11,12)$ and is a major cause of intracerebral hemorrhage (ICH) $(11,13)$. The imbalance of $A \beta$ production and the clearance of $A \beta$ seem to lead to AD-related cognitive impairment (14). Studies have shown that the decrease of endogenous Neprilysin level will prolong the half-life of amyloid beta protein in the body, increase the levels of $\mathrm{A} \beta 40$ and $\mathrm{A} \beta 42$ in brain and plasma, and then lead to the occurrence of amyloid angiopathy (15). Therefore, reducing the aggregation of $A \beta$ or clearing $A \beta$ is an effective therapeutic strategy for CAA. With the aging of the population, there is an urgent need to find a cure for the disease. Studies have shown that the key mechanisms of removal of $A \beta$ include vascular transport across the blood brain barrier (BBB) (16), the glymphatic system (17), enzymatic degradation and cellular uptake, or through the intramural perivascular drainage (IPAD) system $(18,19)$.

CAA mainly arises from $\mathrm{A} \beta$ deposits in the leptomeningeal vessels and penetrating arterioles. The relative incidence of cortical hemorrhage has been increasing, but there is no effective drug treatment for cortical hemorrhage.

In 2005, Conboy et al. (20) proved the regenerative ability of young blood through a parabiosis experiment, in which young and old mice were connected by surgery and shared a blood circulatory system. Then, in 2011, Villeda et al. (21) showed that plasma, rather than whole blood infusion, is the component in young blood that improves the cognitive function of old mice and pointed out that there are soluble factors in old blood that inhibit neurogenesis. Several studies have shown that either by injecting young plasma or by parabiosis, young plasma can reverse synaptic plasticity and cognitive function in older amyloid precursor protein APP transgenic mice (APP mice) (21-23), as well as improving the function of aged organ/tissues, including the liver, brain, and skeletal muscles $(24,25)$. In addition, Sinha et al. (26) found that increasing the circulating protein growth differentiation factor 11 (GDF11) levels in aged mice can regulate muscle aging and improve muscle structure and function. The $\mathrm{C}-\mathrm{C}$ motif chemokine ligand 11 (CCL11) and $\beta 2$-microglobulin levels in mice and humans increase with age, reducing neurogenesis and causing memory impairment in older mice and humans. Their experiments showed that the levels of CCL11 and $\beta 2$-microglobulin in aged mice decreased after young plasma treatment, thus improving the memory impairment of aged mice $(21,27)$. Furthermore, raising testosterone levels can restore skeletal muscle regeneration in aged mice (28). Therefore, several components in young blood play an important role in the brain regeneration of aged mice.

In order to improve the understanding of young blood, 
Wang et al. (29) extracted extracellular vesicles (EVs), from the serum of young mice and evaluated the regeneration effect in old mice. They found that EVs could reduce the chronic inflammatory reaction in old mice. In addition, studies have shown that thrombospondin-4 (THBS 4) and SPARC-like protein 1 (SPARCL1) in the serum of young rats can directly act on neurons and stimulate dendritic formation and synaptic formation (30). Some studies have also shown that cytokines, interleukins, and growth factors secreted by young activated $\mathrm{T}$ lymphocytes significantly increase the proliferation and migration rate of $\mathrm{C} 2 \mathrm{C} 12$ myoblasts, which is beneficial to muscle regeneration (31). Yuan et al. (32) found that young plasma can significantly reduce the acute brain injury caused by cerebral hemorrhage in elderly rodents, and they believe that young plasma and its circulating "young factors", such as IGF-1, may help to treat the symptoms of cerebral hemorrhage in elderly patients. Although young plasma shows beneficial effects in old mice, the effects of young plasma on CAA model mice and the extent to which they are involved in the clearance of $\mathrm{A} \beta$ is not clear.

To determine the effect of young blood plasma on elderly APP mice, we used the young blood injection method by centrifugally separating the blood components of healthy young mice, and then injecting them into the elderly APP/PS1 mice. These APP mice are characterized by the rapid appearance of $A \beta$ depositions (33) and exhibit behavioral phenotypes similar to the cognitive impairment and psychiatric symptoms seen in $\mathrm{AD}$ patients (34). Next, we tested the learning and memory abilities of aged APP/ PS1 mice exposed to young blood. Finally, we analyzed the changes in $A \beta$ expression and the cortical hemorrhage area in the brains of these mice.

We present the following article in accordance with the ARRIVE reporting checklist (available at http://dx.doi. org/10.21037/atm-20-8008).

\section{Methods}

\section{Experimental design}

The following mouse lines were used: young mice ( $\mathrm{n}=50$, aged 3-4 months) and aged APP/PS1 mice (with a C57BL/6J genetic background) ( $\mathrm{n}=10,18-22$ months) were purchased from the Model Animal Research Center of Nanjing University in China (Nanjing, China). Wildtype (WT) mice of the same background (with a C57BL/6J genetic background) and age ( $\mathrm{n}=5,18-22$ months) were used as a control group. All studies were undertaken with female mice. The mice were raised under specific pathogenfree conditions with a 12-hour light-dark cycle. Food and water were freely available. The health status of the mice was monitored every two weeks to exclude problematic mice from the study. The animals were treated according to the standards of animal protection and use according to the Committee of Zhengzhou University. This study was approved by the Ethics Committee of Zhengzhou University.

The mice were assigned to three groups that were equal in size (n=5): WT mice, aged APP/PS1 mice, and aged APP mice plus young plasma (APP/PS1-YP) (Figure 1A). There was no significant difference in body weight and age among the groups.

After the plasma injection phase was completed (28 days), behavioral tests were used to evaluate changes in memory, cognition, and mood of the mice. The $\mathrm{Y}$ maze and new object recognition test (NOR) were conducted on the first day, the open-field test and the forced swimming test were conducted on the second day, the sucrose consumption test was conducted on the third day, and the corner test was conducted on the $1^{\text {st }}, 3^{\text {rd }}$, and $7^{\text {th }}$ days. After the behavioral tests were completed, the mice were anesthetized with $10 \%$ chloral hydrate $(400 \mathrm{mg} / \mathrm{kg})$ and then sacrificed, and the brain tissues were processed for the ELISA and immunofluorescence experiments (Figure 1B).

\section{Blood collection and administration}

The blood of the young mice was taken at the time of death by caudal vein bleeding or intracardiac hemorrhage and collected into an Ethylene Diamine Tetraacetic Acid (EDTA, Shanshan Medical, China) coated tube. The whole blood was used for centrifugation at $1,000 \mathrm{~g}$ for 10 minutes at $4{ }^{\circ} \mathrm{C}$. Then the plasma and lymphocyte aliquots were stored at $-80^{\circ} \mathrm{C}$ until use. Before administration, the plasma was dialyzed with a $3.5 \mathrm{kDa}$ Slide-A-lyzer dialyzer (Thermo Scientific, USA) in a phosphate buffered solution (PBS) to remove the EDTA. The mice in the treatment group were systematically treated by injecting young plasma $(100 \mu \mathrm{L} /$ injection) into the tail vein twice a week for 4 weeks.

\section{Behavior testing}

\section{Y-maze}

The Y-maze test was used to assess the short-term memory of the mice. The maze consisted of three $30 \mathrm{~cm}$-long arms 


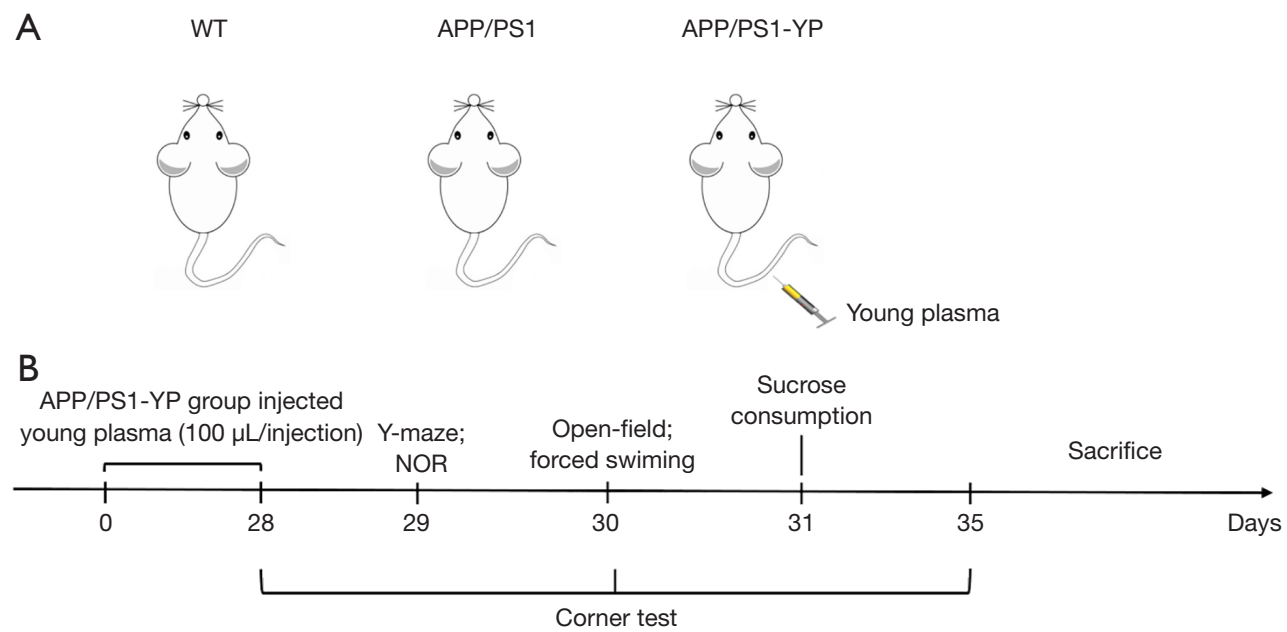

Figure 1 Experimental design. (A) The experiment was divided into three groups, in which APP/PS1-YP mice were injected with young plasma (100 L/injection) through the tail vein twice a week for 4 weeks; (B) after completing various behavioral tests, the mice were sacrificed. WT, wild-type mice; APP/PS1, APP/PS1 mice; APP/PS1-YP, aged APP/PS1 mice plus young plasma; NOR, novel object recognition.

(named starter arm, novel arm and other arm) at a $120^{\circ}$ angle to each other in the shape of a capital $Y$ and was made of grey wood covered with black paper. The mouse was placed on the distal end of the starting arm, the novel arm was blocked with a partition, and the mouse was allowed to explore freely in the maze for 5 minutes. The maze was then wiped with $80 \%$ alcohol to prevent odor hints. Two hours later, the novel arm was opened, and the mouse was put into the Y-maze again to evaluate its spatial memory. The number of times and time the mouse entered the novel arm was recorded, as well as the total exploration time.

\section{NOR test}

The novel object recognition test consists of the habit phase, training phase and testing phase. Before the experiment, each mouse was put in a black polyethylene plastic test box $(25 \mathrm{~cm} \times 40 \mathrm{~cm} \times 25 \mathrm{~cm})$ to adapt for 48 hours. After 24 hours, two identical objects were placed at the opposite corners of the box. The mouse was placed in the test box for 10 minutes before the test in order to become comfortable with the equipment. Then the mouse was placed back in its cage and the objects and boxes were cleaned between each animal with $80 \%$ ethanol to reduce the smell that might affect the results of the next mouse test. An hour later, one of the objects was replaced by a novel, different object. Again, each mouse was given 3 minutes to explore the object. The mice were considered to be exploring the novel object only if they were within
$2 \mathrm{~cm}$ of the novel object and not sitting on or leaning against it. The exploration times of the novel object and the familiar object were recorded, and the discrimination index (DI) was used to evaluate the mouse's recognition ability, that is, DI = (time exploring novel object)/(time exploring novel object + time exploring familiar object) $\times 100 \%$.

\section{Forced swimming test}

In the forced swimming test, the mice were placed in containers containing warm water $\left(23-25^{\circ} \mathrm{C}\right)$ for 8 minutes (width: $20 \mathrm{~cm}$; length: $40 \mathrm{~cm}$; depth: $20 \mathrm{~cm}$; water level: $14-15 \mathrm{~cm})$. The mice were not allowed to touch the bottom of the container. The duration of immobility was recorded during the last 6 minutes of the 8 -minute test period. When the mouse floated in an upright position it was considered immobile, and mice with depressive-like behavior showed increased immobile time.

\section{Open field test}

The open field test was used to evaluate the exploratory activity and anxiety behavior of the mice. It consisted of a $50 \mathrm{~cm} \times 50 \mathrm{~cm} \times 40 \mathrm{~cm}$ wooden box, with a center area of $25 \mathrm{~cm} \times 25 \mathrm{~cm}$ and the remaining surrounding area. Each mouse was placed in the center of the field and allowed to explore freely for 10 minutes in a quiet environment. Then the field was cleaned with $50 \%$ alcohol to remove the scent marks. The distance covered and amount of time spent in the center and surrounding areas were measured by the 
tracking system. The percentage of time the mouse spent in the center and corner was calculated as a measure of anxiety.

\section{Sucrose consumption}

Animals tend to ingest large amounts of sweet substances or solutions. Compared with their normal daily liquid intake, rodents show a significantly higher preference for sucrose solution and consume more liquid when sucrose solution is introduced. However, the sucrose intake of depressed mice is known to decrease. In this study, the mice were subjected to 3 hours of food and water deprivation before the sucrose test. Then, the same amount of ordinary water with a $2 \%$ sucrose solution was placed in a cage familiar to the mice to avoid the effects of the external environment on the results. The amount of normal water with a $2 \%$ sucrose solution consumed within 3 hours by each animal was recorded. The results were expressed as sucrose consumption per animal $(\mathrm{mL}) /($ water consumption + sucrose consumption $) \times 100 \%$.

\section{Corner test}

The corner test is used to test sensory and motor disorders after cerebral cortex and substantia nigra striatum injury at 1,7 and 14 days after cerebral hemorrhage. The experimental device consists of two boards each measuring $30 \mathrm{~cm} \times 20 \mathrm{~cm} \times 1 \mathrm{~cm}$ with the edges of the boards connected at an angle of 30 degrees. The mouse is placed between the two boards. When the mouse enters the depths of the corner, the whiskers on both sides are stimulated. Normally, the mouse will turn upward, left or right, and then leave the corner. Mice affected by cerebral ischemia tend to turn to the undamaged side (35). In this study, this process was repeated for each test until 10 correct turns were recorded for each animal and the percentage of left turns was calculated. Occasional turns were not counted.

\section{Enzyme-linked immunosorbent assay (ELISA)}

A beta-amyloid total A $\beta$ ELISA Kit (Covance, Princeton, NJ, USA), was used according to the manufacturer's instructions to measure the level of soluble $A \beta$ in the brains and peripheral blood of the mice. The hippocampus of each group was homogenized in lysis buffer. Diluted protein standards and samples were added to a 96-well ELISA plate, and then HRP-labeled antibodies for A $\beta$ were added to form an antibody-antigen-enzyme-labeled antibody complex. After washing with phosphate buffered saline (PBS) solution, a solution of TMB substrate was added and converted to blue under the catalysis of HRP. Then the stop solution was added to stop the reaction. The absorbance at $450 \mathrm{~nm}$ was detected by a microplate reader, and the concentration of $\mathrm{A} \beta$ was calculated according to the standard curve.

\section{Immunofuorescence}

The mice were anesthetized with $400 \mathrm{mg} / \mathrm{kg}$ chloral hydrate (Sigma-Aldrich, USA), then $0.9 \%$ saline was infused into the hearts of the mice, and their brains were dissected and fixed in $4 \%$ paraformaldehyde phosphate buffer at $4{ }^{\circ} \mathrm{C}$ for 48 hours, and then cryopreserved with $30 \%$ sucrose. A cryostat was used to cut $30 \mu \mathrm{m}$ continuous sections. Next, the sections were incubated with $0.3 \%$ hydrogen peroxide in methanol for 10 minutes, and then blocked with $10 \%$ normal goat serum in PBS for 20 minutes. Single staining of the IBA1 (ionized calcium binding adaptor molecule 1) and GFAP (glial fibrillary acidic protein), was achieved by blocking with anti-Iba1 (1:200, goat polyclonal antiIba1, Abcam, USA, \#ab5076) and anti-GFAP (1:2,500, chicken polyclonal ant-GFAP, Abcam, USA, \#ab4674) and incubated at $4{ }^{\circ} \mathrm{C}$ overnight in the solution. After washing three times with PBS the next day, the sections were incubated with an Alexa 484-conjugated IgG secondary antibody (Invitrogen, CA, USA) for 2 hours, and finally 4',6-diamino-2-phenylindole (DAPI) (Solarbio, China) was used for the fluorescent nuclear staining. After 15 minutes of incubation, the coverslip was thoroughly washed with distilled water.

\section{Immunofluorescence analysis and quantification}

A confocal scanning microscope (Leica, Germany) was used to image the immunofluorescence staining. The ImageJ software of the National Institutes of Health was used to measure the average signal intensity. The intensities of Iba1 and GFAP were measured in the whole image. The hippocampus and hemispherical cortex of each group of animals were analyzed. Then the average area of Iba1 positive cells was measured by ImageJ software, and the quantity of GFAP positive cells was expressed as a percentage.

\section{Statistical analysis}

The data are expressed as mean \pm SD. The computer program GraphPad Prism 8.0 (Graph-Pad Software, 
Inc., La Jolla, CA, USA) was used for all analyses, with a statistical significance level of $\mathrm{P}<0.05$. The ShapiroWilk test or Kolmogorov-Smirnov test was used to test the normality of the data distribution in each group of experiments. The $\mathrm{F}$ test showed that there was no significant difference in variance among groups. One-way ANOVA was used to analyze between-group differences, and a two-way ANOVA was used to analyze the corner test data followed by a post hoc Tukey's test.

\section{Results}

\section{Young plasma improves memory, cognition, and mood in APP/PS1 mice}

To evaluate whether young plasma can improve cognitive function and emotion in APP/PS1 mice, we injected young plasma into APP/PS1 mice, and then carried out several behavioral tests. In the Y maze test of spatial working memory, the number of times and time spent entering the new arm of APP/PS1-YP were significantly higher than those of APP/PS1 mice (Figure $2 A, B,{ }^{*} \mathrm{P}<0.05$, $\left.{ }^{* *} \mathrm{P}<0.01,{ }^{* * * *} \mathrm{P}<0.0001\right)$, indicating that young plasma improved the short-term memory of APP/PS1 mice. In order to examine the effect of young plasma on recognition memory, we carried out the NOR test. One-way analysis of variance (ANOVA) showed that $\mathrm{F}=48.47, \mathrm{P}<0.0001$. The discrimination index of APP/PS1 mice was significantly lower than that of normal WT mice, and the discrimination index of APP/PS1-YP was significantly higher than that of APP/PS1 mice (Figure 2C). The above data suggest that young plasma can effectively improve the cognitive and memory function of APP/PS1 mice.

The forced swimming test was used to assess the effect of youth plasma on anxiety in APP/PS1 mice. In comparison with the control group, the immobile time of the APP/ PS1 group and the APP/PS1-YP group was increased. However, the immobile time of the APP/PS1-YP group was significantly shorter than that of the APP/PS1 group. (ANOVA, Figure 2D). In the sucrose consumption test, the result of the Brown-Forsythe ANOVA was $\mathrm{F}_{(2,10.65)}=27.74$ $\mathrm{P}<0.0001$. Games-Howell's multiple comparisons test showed that the sucrose consumption ratio of the APP/PS1 group was significantly lower than that of the WT group $\left({ }^{* *} \mathrm{P}<0.001\right.$, Figure $\left.2 E\right)$, while the APP/PS1-YP group's consumption was significantly higher than that of the APP/PS1 group $\left({ }^{* *} \mathrm{P}<0.01\right.$, Figure $\left.2 E\right)$. It is suggested that young plasma therapy partially restored the depression-like behavior of the APP/PS1 mice.

Figure $2 F, \mathrm{G}$ detail the results of the open field test. Compared with the control group, the central exploration time of the APP/PS1 mice was significantly lower ${ }^{* *} \mathrm{P}<0.01$, Figure $2 F$ ), and the ratio of central exploration time to total exploration time was also significantly lower than that of the control group $\left({ }^{*} \mathrm{P}<0.05\right.$, Figure $\left.2 G\right)$. However, compared with the APP/PS1 mice, the APP/PS1-YP mice spent a longer time in the center $\left({ }^{*} \mathrm{P}<0.05\right)$ and had a higher ratio to total exploration time $\left({ }^{*} \mathrm{P}<0.05\right)$. This test indicates that young plasma can effectively improve the anxiety of APP/ PS1 mice.

The corner test (Figure $2 H$ ) two-way ANOVA showed an interaction effect of $\mathrm{F}_{(4,36)}=7.323 \mathrm{P}<0.001$. Tukey's multiple comparisons test indicated that the percentage of left turns in the APP groups was significantly lower than that in the WT group $\left({ }^{* * * *} \mathrm{P}<0.0001\right)$, while that in the APP/PS1-YP group was significantly higher than that in APP/PS1 group $\left({ }^{* *} \mathrm{P}<0.01\right)$.

\section{Young plasma promotes neurogenesis in APP/PS1 mice}

To determine whether young plasma can increase neurogenesis in APP/PS 1 mice, we performed an immunofluorescence analysis (Figure $3 A$ ). The results showed that the number of neurons in the APP/PS1 mice were significantly increased compared with those of the APP/PS1 mice treated with young plasma (Figure 3B,C). These results indicate that the decrease in neurogenesis in APP/PS1 mice is at least partially restored by treatment with young plasma.

\section{Young plasma does not significantly reduce Aß levels in APP/PS1 mice}

We first analyzed the levels of $\mathrm{A} \beta 40$ and $\mathrm{A} \beta 42$ in the total homogenates of the cortex and hippocampus of the three groups of aged mice by ELISA (Figure 3D,E). The data showed that compared with the APP/PS1 group, the levels of $A \beta_{40}$ and $A \beta_{42}$ in the cortex and hippocampus of the APP/ PS1-YP group did not change significantly. This indicates that injection of young plasma does not reduce $A \beta$ levels in the cortex and hippocampus.

\section{Young plasma can reduce the cortical hemorrhage area in APP/PS1 mice}

We explored the condition of ICH in the cerebral cortex of 
A

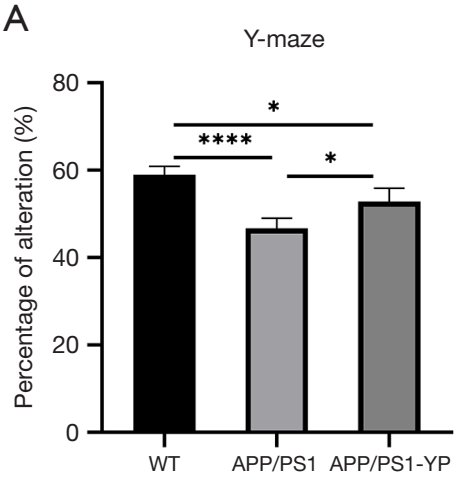

D

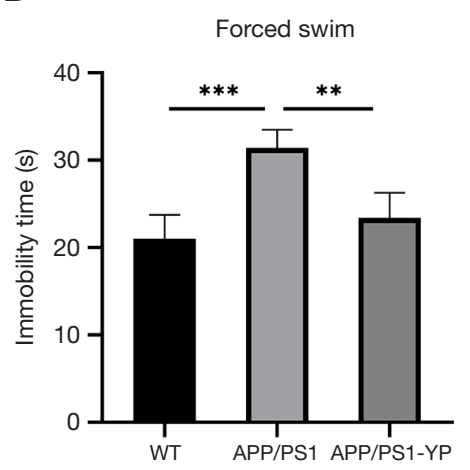

G

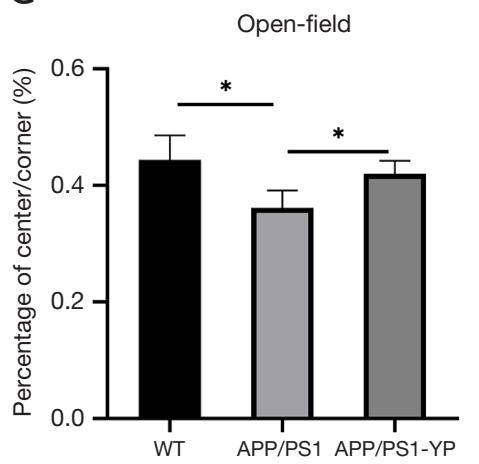

B

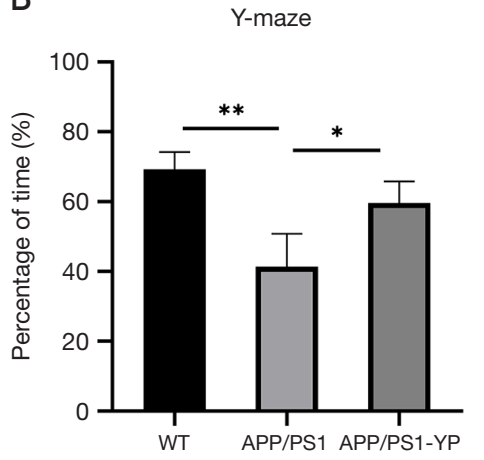

E

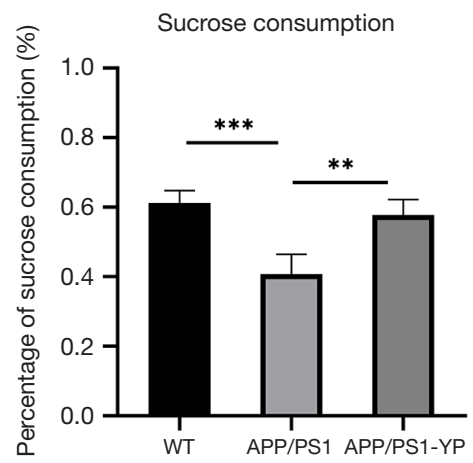

$\mathrm{H}$

(1)

क्ष

Corner test
C

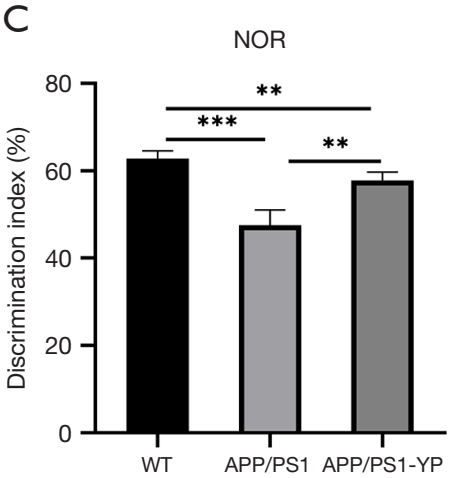

$\mathrm{F}$

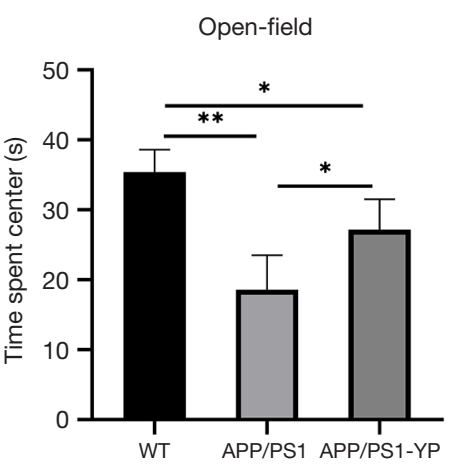

A

- $1 \mathrm{~d}$
$\square 3 \mathrm{~d}$
$\square 7 \mathrm{~d}$

Figure 2 The changes in cognition, memory and emotional behavior of mice in each group. (A) In the Y-maze, the ratio of the time spent on the new arm to the total time was recorded. (B) The percentage of times the mice entered the new arm. (C) In the new object recognition experiment, the percentage of the mouse's exploration time on novel objects in the total exploration time. (D) In the forced swimming test, the duration of immobility in the container was recorded, and the mice with depressive behavior stayed longer. (E) The percentage of $2 \%$ sucrose solution consumed by mice within 3 hours. (F) The duration of time mice stayed in the center was recorded in the open-field test. (G) The time ratio of center to corner in the open field test. $(\mathrm{H})$ The percentage of mice turning left in the corner test. Results are mean $\pm \mathrm{SD}$. Statistics: ${ }^{*} \mathrm{P}<0.05 ;{ }^{* *} \mathrm{P}<0.01 ;{ }^{* * *} \mathrm{P}<0.001 ;{ }^{* * * *} \mathrm{P}<0.0001$. WT, wild-type mice; APP/PS1, APP/PS1 mice; APP/PS1-YP, aged APP/PS1 mice plus young plasma; NOR, novel object recognition. 


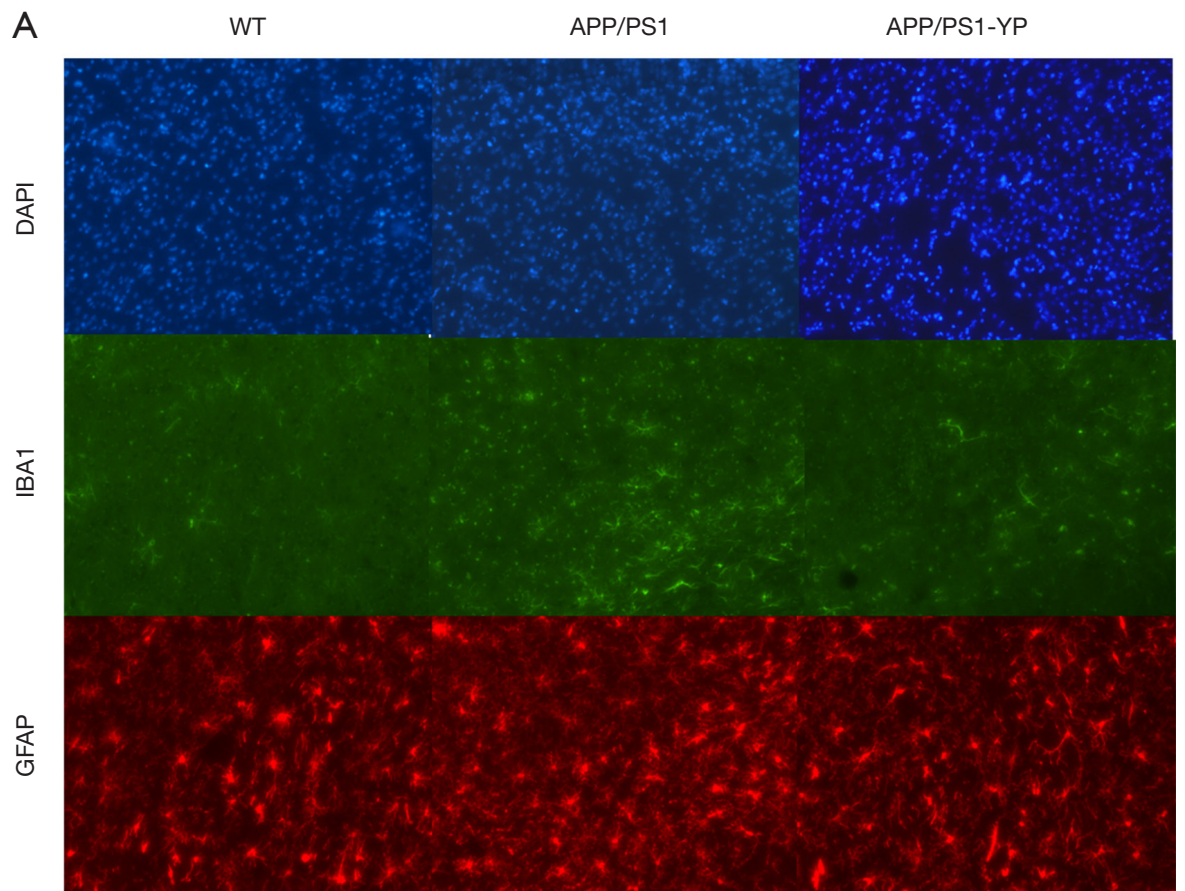

B

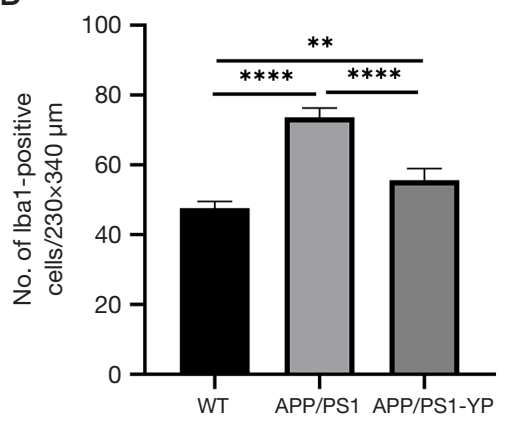

D

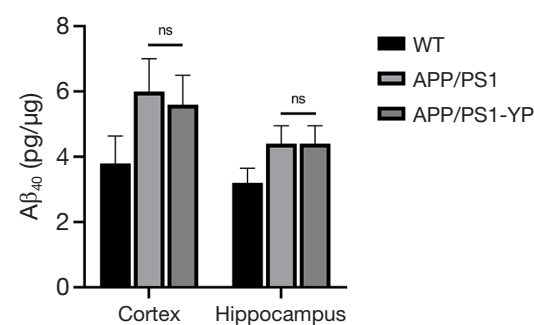

C

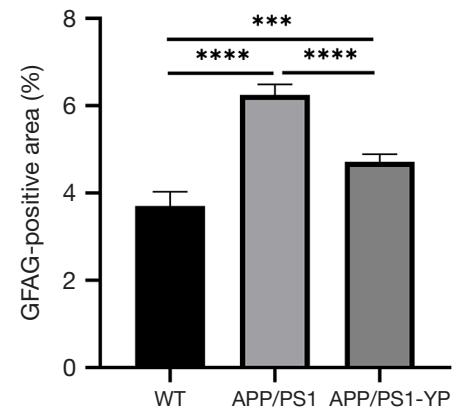

$\mathrm{E}$

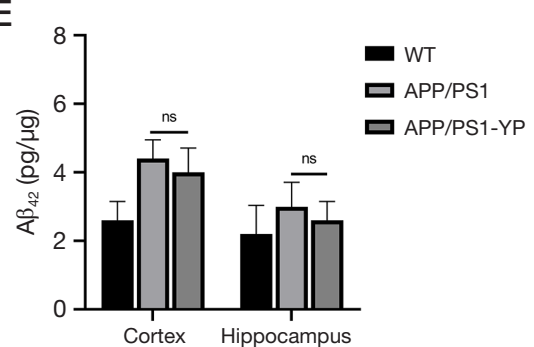

Figure 3 Immunofluorescence and ELISA analysis. (A) Representative areas of Iba1 and GFAP positive cells, Scale bar = $50 \mu$ m; (B) average area of Iba1 positive cells; (C) quantitative percentage of GFAP positive cells; (D) ELISA analysis of A 40 levels in mouse cerebral cortex and hippocampus; (E) ELISA analysis of A $\beta 42$ levels in mouse cerebral cortex and hippocampus; Results are mean $\pm \mathrm{SD}$. Statistics: ${ }^{* *} \mathrm{P}<0.01$; ${ }^{* * *} \mathrm{P}<0.001 ;{ }^{* * * *} \mathrm{P}<0.0001$. WT, wild-type mice; APP/PS1, APP/PS1 mice; APP/PS1-YP, aged APP/PS1 mice plus young plasma; NOR, novel object recognition; IBA1, ionized calcium binding adaptor molecule 1; GFAP, glial fibrillary acidic protein; DAPI, 6-diamino-2phenylindole. 
A
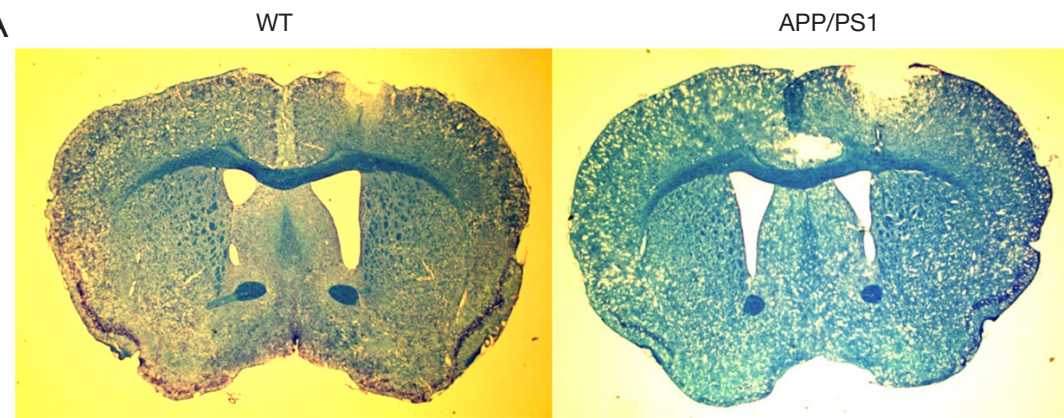

APP/PS1-YP

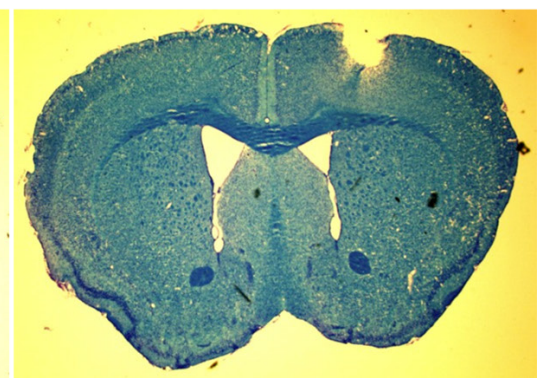

B

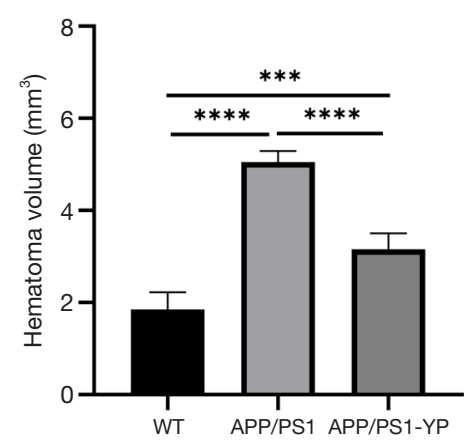

Figure 4 The representative fast blue-stained sections and the quantitative results of hematoma volume were obtained in each group. (A) Representative fast blue-stained sections of the three groups of mice. Scale bar $=1 \mathrm{~mm}$. (B) Quantitative results of hematoma volume based on fast blue-stained sections. ${ }^{* *} \mathrm{P}<0.001 ;{ }^{* * *} \mathrm{P}<0.0001$. WT, wild-type mice; APP/PS1, APP/PS1 mice; APP/PS1-YP, aged APP/PS1 mice plus young plasma.

mice. The brain tissues of the different groups of mice were stained with fast blue to estimate the size of the hematoma, and then the cortical hemorrhage area of each group of mice was measured. Overall, the hematoma area of the WT group was smaller than that of the APP/PS1 group, while the hematoma area of the APP/PS1 group was larger than that of the APP/PS1-YP group (Figure $4,{ }^{* * * *} \mathrm{P}<0.0001$ ). This data indicates that young plasma can reduce the cortical hemorrhage area of APP/PS1 mice.

\section{Discussion}

In summary, our research shows that injecting young plasma into APP/PS1 mice not only significantly improves the cognitive memory and anxiety of APP/PS1 mice, but also reduces the area of cerebral cortex hemorrhage caused by CAA disease. However, treatment with young plasma did not reduce amyloid levels in late-stage mice. This study further confirmed the positive and beneficial effects of young plasma on CAA disease.

Our experimental results showed that there was no significant decrease in the level of $A \beta$ in the brain and peripheral blood of elderly mice infused with young plasma. A series of studies have shown that the reduction of the synaptic proteins synaptophysin and calbindin in the dentate gyrus in $\mathrm{AD}$ mouse models was reversed by heterochronic parabiosis experiment, but the levels of amyloid plaques, soluble $A \beta$ and $A \beta_{42}$, and the activation of microglia were not changed (36). In old mice, young mouse plasma had a good effect on some disease parameters but it did not reverse all aspects, insofar as treatment with young plasma did not reduce the level of amyloid protein in the mouse model (37). Furthermore, the process of blood circulation from young mice to $\mathrm{AD}$ mice reversed the loss of hippocampal synapses and the abnormal expression of many genes involved in key neuronal signal pathways but did not reduce the deposition of $A \beta$ or the activation of microglia (38).

Young blood reverses cognitive deficits in aged mice. The presence of one or more soluble $A \beta$ aggregates may cause some early morphological and functional synaptic defects, which may lead to memory dysfunction in APP mice (39). Consistent with our findings, Villeda et al. (21) have shown that aged mice that share blood with young 
mice exhibit enhanced long-term potentiation (LTP)increased neurogenesis and improved cognitive function. Other studies have shown that injecting young plasma into old mice can improve their performance on the Radial Arm Water Maze (RAWM) test, novel object recognition tests and contextual fear conditioning (40). This was further expanded by the use of human plasma extracted from the umbilical cord. Human cord plasma therapy revitalized the hippocampus and enhanced synaptic plasticity and hippocampal-dependent cognitive ability in aged mice (41). These results support a role for young plasma in slowing down the progression of diseases associated with amyloid deposition.

However, some studies have suggested that young plasma can reduce the pathological changes of hippocampal $\mathrm{A} \beta$ plaques. Xia et al. (42) have shown that intravenous injection of young serum can alleviate the learning and memory impairment of aged $\mathrm{AD}$ model mice, reduce the pathological changes of hippocampal A $\beta$ plaques, restore synaptic formation and synaptic plasticity, and then repair hippocampal cholinergic circuits. Zhao et al. (43) also believe that young plasma therapy can not only reduce neuroinflammation in the brain of $\mathrm{AD}$ model mice, but also reduce the pathological levels of tau and $\mathrm{A} \beta$. We believe that the reason for the inconsistency between our research and these findings may be related to the duration, frequency, and plasma dose of young blood injections. At present, the most suitable time, frequency and dose of continuous injection of young blood have not been determined. Since a large number of studies have demonstrated the potential therapeutic characteristics of young blood for elderly diseases, it is necessary to further clarify the continuous injection time, frequency, duration, and plasma dose of young blood.

In this study, we studied the effects of young plasma therapy on behavior and brain A $\beta$ protein levels in CAA model mice. Considering that young plasma may also have effects on blood lipids, amyloid plaque deposition, neuroinflammatory proteins and mRNA markers, we will evaluate them in the next study, which will be reflected in our follow-up research articles.

Although young plasma shows beneficial effects in the treatment of APP mice, this is only a preclinical animal study, and whether it also shows beneficial effects in human diseases needs to be confirmed by larger clinical trials. Moreover, the effect of the mouse model used in this study on the frequency of plasma administration or the volume given per injection have yet to be studied. However, this study is important because it demonstrates the potential therapeutic value of young plasma by improving the symptoms of diseases associated with $A \beta$ deposition. At present, no harmful effects of young plasma on aged APP mice have been found.

In summary, young blood plays an important role in human neurodegenerative and neurovascular diseases and shows promise for a potential clinical application. Our study provides further experimental evidence to confirm the potential role of young plasma in the treatment of patients with CAA. It is not fully clear how young plasma exerts its effect. Future studies must clarify the mechanisms of these interactions in order to determine their potential in therapeutic applications.

\section{Conclusions}

In summary, we found that young plasma did not reduce amyloidosis in a CAA mouse model, but young plasma improved cognitive impairment and cortical hemorrhage in CAA model mice, and no adverse effects of young plasma were observed during the experiment. Our study shows that young plasma is promising as a clinical therapeutic agent for CAA.

\section{Acknowledgments}

Funding: Youth Project of National Natural Science Foundation of China, 81701162.

\section{Footnote}

Reporting Checklist: The authors have completed the ARRIVE reporting checklist. Available at http://dx.doi. org/10.21037/atm-20-8008

Data Sharing Statement: Available at http://dx.doi. org/10.21037/atm-20-8008

Conflicts of Interest: All authors have completed the ICMJE uniform disclosure form (available at http://dx.doi. org/10.21037/atm-20-8008). The authors have no conflicts of interest to declare.

Ethical Statement: The authors are accountable for all aspects of the work in ensuring that questions related to the accuracy or integrity of any part of the work are appropriately investigated and resolved. The animals were 
treated according to the standards of animal protection and use according to the Committee of Zhengzhou University. This study was approved by the Ethics Committee of Zhengzhou University.

Open Access Statement: This is an Open Access article distributed in accordance with the Creative Commons Attribution-NonCommercial-NoDerivs 4.0 International License (CC BY-NC-ND 4.0), which permits the noncommercial replication and distribution of the article with the strict proviso that no changes or edits are made and the original work is properly cited (including links to both the formal publication through the relevant DOI and the license). See: https://creativecommons.org/licenses/by-nc-nd/4.0/.

\section{References}

1. Vonsattel JP, Myers RH, Hedley-Whyte ET, et al. Cerebral amyloid angiopathy without and with cerebral hemorrhages: a comparative histological study. Ann Neurol 1991;30:637-49.

2. Greenberg SM, Vonsattel JP, Stakes JW, et al. The clinical spectrum of cerebral amyloid angiopathy: presentations without lobar hemorrhage. Neurology 1993;43:2073-9.

3. Knudsen KA, Rosand J, Karluk D, et al. Clinical diagnosis of cerebral amyloid angiopathy: validation of the Boston criteria. Neurology 2001;56:537-9.

4. Greenberg SM, Gurol ME, Rosand J, et al. Amyloid angiopathy-related vascular cognitive impairment. Stroke 2004;35:2616-9.

5. ChaoCP, Kotsenas AL, Broderick DF. Cerebral amyloid angiopathy: $\mathrm{CT}$ and MR imaging findings. Radiographics 2006;26:1517-31.

6. Verbeek MM, Kremer BP, Rikkert MO, et al. Cerebrospinal luid amyloid beta(40)is decreased in cerebral amyloid angiopathy. Ann Neurol 2009;66:245-249.

7. Rannikmäe K, Kalaria RN, Greenberg SM, et al. APOE associations with severe CAA-associated vasculopathic changes: Collaborative meta-analysis. J Neurol Neurosurg Psychiatry 2014;85:300-5.

8. Van Rooden S, van der Grond J, Van den Boom R, et al. Descriptive analysis of the Boston criteria applied to a Dutchtype cerebral amyloid angiopathy population. Stroke 2009;40:3022-7.

9. Jellinger KA. Alzheimer disease and cerebrovascular pathology: an update. J Neural Transm (Vienna) 2002;109:813-36.

10. Lin B, Hasegawa Y, Takane K, et al. High-Fat-Diet Intake
Enhances Cerebral Amyloid Angiopathy and Cognitive Impairment in a Mouse Model of Alzheimer's Disease, Independently of Metabolic Disorders. J Am Heart Assoc 2016;5:e03154.

11. Charidimou A, Boulouis G, Gurol ME, et al. Emerging concepts in sporadic cerebral amyloid angiopathy. Brain 2017;140:1829-50.

12. Vinters HV. Cerebral amyloid angiopathy. A critical review. Stroke 1987;18:311-24.

13. Charidimou A, Gang Q, Werring DJ. Sporadic cerebral amyloid angiopathy revisited: recent insights into pathophysiology and clinical spectrum. J Neurol Neurosurg Psychiatry 2012;83:124-37.

14. Hardy J, Selkoe DJ. The amyloid hypothesis of Alzheimer's disease: progress and problems on the road to therapeutics. Science 2002;297:353-6.

15. Farris W, Schutz S G, Cirrito J R, et al. Loss of neprilysin function promotes amyloid plaque formation and causes cerebral amyloid angiopathy. Am J Pathol 2007;171:241-51.

16. Shibata M, Yamada S, Kumar SR, et al. Clearance of Alzheimer's amyloid-ss(1-40) peptide from brain by LDL receptor-related protein-1 at the blood-brain barrier. J Clin Invest 2000;106:1489-99.

17. Iliff JJ, Wang M, Liao Y, et al. A paravascular pathway facilitates CSF flow through the brain parenchyma and the clearance of interstitial solutes, including amyloid beta. Sci Transl Med 2012;4:147ra11.

18. Preston SD, Steart PV, Wilkinson A, et al. Capillary and arterial cerebral amyloid angiopathy in Alzheimer's disease: defining the perivascular route for the elimination of amyloid beta from the human brain. Neuropathol Appl Neurobiol 2003;29:106-17.

19. Weller RO, Massey A, Newman TA, et al. Cerebral amyloid angiopathy: amyloid beta accumulates in putative interstitial fluid drainage pathways in Alzheimer's disease. Am J Pathol 1998;153:725-33.

20. Conboy IM, Conboy MJ, Wagers AJ, et al. Rejuvenation of aged progenitor cells by exposure to a young systemic environment. Nature 2005;433:760-4.

21. Villeda SA, Luo J, Mosher KI, et al. The ageing systemic milieu negatively regulates neurogenesis and cognitive function. Nature 2011;477:90-4.

22. Villeda SA, Plambeck KE, Middeldorp J, et al. Young blood reverses age-related impairments in cognitive function and synaptic plasticity in mice. Nat Med 2014;20:659-63.

23. Katsimpardi L, Litterman NK, Schein PA, et al. Vascular and neurogenic rejuvenation of the aging mouse brain by 
young systemic factors. Science 2014;344:630-4.

24. Castellano JM, Kirby ED, Wyss-Coray T. BloodBorne Revitalization of the Aged Brain. JAMA Neurol 2015;72:1191-4.

25. Wyss-Coray T. Ageing, neurodegeneration and brain rejuvenation. Nature 2016;539:180-6.

26. Sinha M, Jang YC, Oh J, et al. Restoring systemic GDF11 levels reverses age-related dysfunction in mouse skeletal muscle. Science 2014;344:649-52.

27. Smith LK, He Y, Park JS, et al. beta2-microglobulin is a systemic pro-aging factor that impairs cognitive function and neurogenesis. Nat Med 2015;21:932-7.

28. Sinha I, Sinha-Hikim AP, Wagers AJ, et al. Testosterone is essential for skeletal muscle growth in aged mice in a heterochronic parabiosis model. Cell Tissue Res 2014;357:815-21.

29. Wang W, Wang L, Ruan L, et al. Extracellular vesicles extracted from young donor serum attenuate inflammaging via partially rejuvenating aged $\mathrm{T}$-cell immunotolerance. FASEB J 2018;32:fj201800059R.

30. Gan KJ, Sudhof TC. Specific factors in blood from young but not old mice directly promote synapse formation and NMDA-receptor recruitment. Proc Natl Acad Sci U S A 2019;116:12524-33.

31. Al-Dabbagh S, McPhee JS, Murgatroyd C, et al. The lymphocyte secretome from young adults enhances skeletal muscle proliferation and migration, but effects are attenuated in the secretome of older adults. Physiol Rep 2015;3:e12518.

32. Yuan JJ, Zhang Q, Gong CX, et al. Young plasma ameliorates aging-related acute brain injury after intracerebral hemorrhage. Biosci Rep 2019;39: BSR20190537.

33. Rockenstein E, Mallory M, Mante M, et al. Early formation of mature amyloid-beta protein deposits in a mutant APP transgenic model depends on levels of Abeta(1-42). J Neurosci Res 2001;66:573-82.

Cite this article as: Li N, Zhang $\mathrm{X}, \mathrm{Gu}$ Z, Su C, Lian H. Young plasma attenuates cognitive impairment and the cortical hemorrhage area in cerebral amyloid angiopathy model mice. Ann Transl Med 2021;9(2):147. doi: 10.21037/atm-20-8008
34. Faizi M, Bader PL, Saw N, et al. Thy1-hAPP(Lond/ Swe+) mouse model of Alzheimer's disease displays broad behavioral deficits in sensorimotor, cognitive and social function. Brain Behav 2012;2:142-54.

35. Zhang L, Schallert T, Zhang ZG, et al. A test for detecting long-term sensorimotor dysfunction in the mouse after focal cerebral ischemia. J Neurosci Methods 2002;117:207-14.

36. Aicardi G. Young Blood Plasma Administration to Fight Alzheimer's Disease? Rejuvenation Res 2018;21:178-81.

37. Middeldorp J, Lehallier B, Villeda SA, et al. Preclinical Assessment of Young Blood Plasma for Alzheimer Disease. JAMA Neurol 2016;73:1325-33.

38. Shetty AK, Kodali M, Upadhya R, et al. Emerging AntiAging Strategies - Scientific Basis and Efficacy. Aging Dis 2018;9:1165-84.

39. Jacobsen JS, Wu CC, Redwine JM, et al. Early-onset behavioral and synaptic deficits in a mouse model of Alzheimer's disease. Proc Natl Acad Sci U S A 2006;103:5161-6.

40. Khrimian L, Obri A, Ramos-Brossier M, et al. Gpr158 mediates osteocalcin's regulation of cognition. J Exp Med 2017;214:2859-73.

41. Castellano JM, Mosher KI, Abbey RJ, et al. Human umbilical cord plasma proteins revitalize hippocampal function in aged mice. Nature 2017;544:488-92.

42. Xia E, Xu F, Hu C, et al. Young Blood Rescues the Cognition of Alzheimer's Model Mice by Restoring the Hippocampal Cholinergic Circuit. Neuroscience 2019;417:57-69.

43. Zhao Y, Qian R, Zhang J, et al. Young blood plasma reduces Alzheimer's disease-like brain pathologies and ameliorates cognitive impairment in $3 \times \mathrm{Tg}-\mathrm{AD}$ mice. Alzheimers Res Ther 2020;12:70.

(English Language Editor: D. Fitzgerald) 